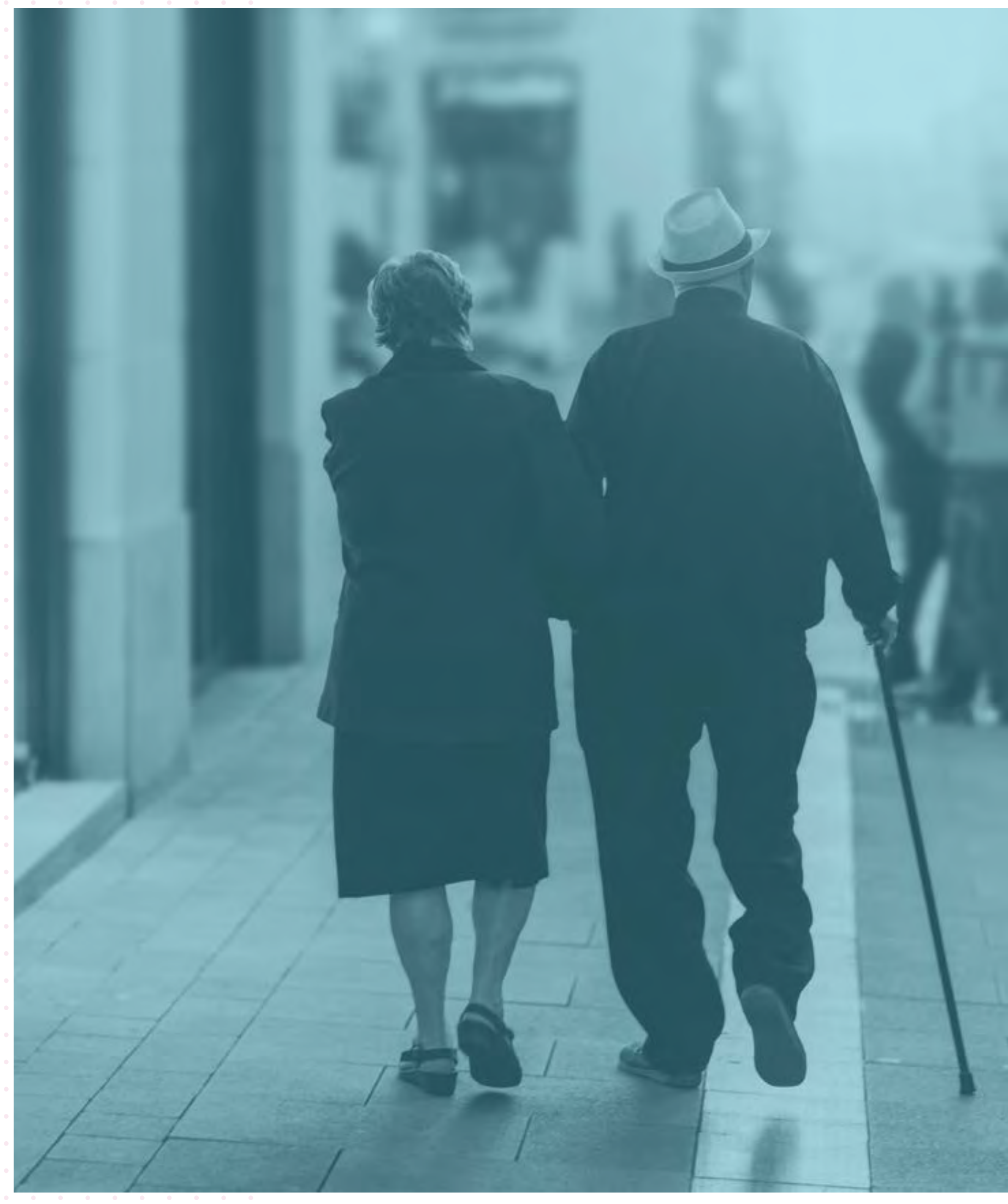




\section{La constitucionalización de los derechos de las personas mayores: Una práctica emergente}

Frederic Megret ${ }^{237}$ y Alexander Agnello 238

DOI: 10.53110/MAWX8628

\section{A. Introducción: Las personas mayores son tratadas explicita-} mente en las constituciones de al menos 106 Estados miembros de la ONU (Anexo 1). Las cuestiones relacionadas con las personas de edad se consideran claramente una preocupación pública que merece un tratamiento constitucional. Sin embargo, las constituciones nacionales, la mayoría de las veces, enmarcan estos temas en términos estrictos de salud o económicos; tienden a concentrarse únicamente en brindar a las personas mayores servicios médicos especializados o seguridad social, como si la vejez fuera una deficiencia que requiriera soluciones útiles.

Por el contrario, centramos nuestro análisis en la necesidad de disposiciones constitucionales que, en general, sean más habilitantes de las personas adultas mayores mediante la protección de sus derechos. Las disposiciones que son habilitantes de derechos humanos parten del estrecho enfoque asistencialista anterior, y en cambio, les empoderan para vivir una

237 Profesor titular y becario William Dawson en la Facultad de Derecho, y codirector del Centro de Derechos Humanos y Pluralismo Jurídico de la Facultad de Derecho de la Universidad McGill. De 2006 a 2016 ocupó la Cátedra de Investigación de Canadá sobre Derechos Humanos y Pluralismo Legal. Antes de llegar a McGill, fue profesor asistente en la Universidad de Toronto, investigador asociado en el Instituto Universitario Europeo y agregado en el Comité Internacional de la Cruz Roja.

238 Investigador y candidato a Maestría en Derecho (LL.M.) en la Facultad de Derecho McGill, donde también obtuvo los títulos de Juris Doctor (J.D.) y Licenciatura en Derecho Civil (B.C.L.). Ha trabajado para la OCDE, el Banco Asiático de Desarrollo y el Defensor del Pueblo de Columbia Británica. 
vida digna mediante el desarrollo de sus capacidades en formas responsivas a sus necesidades y experiencias específicas. El enfoque que recomendamos, en otras palabras, es similar a la transición en el discurso de los derechos de las personas con discapacidad, por ejemplo, entre un modelo asistencialista/médico y uno basado en la dignidad/derechos.

La investigación que condujo a este capítulo identificó tales disposiciones en 29 constituciones nacionales (Anexo 2), disposiciones que ayudan a mostrar cómo la experiencia de las personas mayores está al menos tan construida por factores sociales, económicos y políticos como por el mero hecho de la vejez. ${ }^{239}$ Estas disposiciones garantizan a las personas mayores una amplia gama de derechos en formas que, afirmamos, hacen justicia a la complejidad de su existencia: el derecho a la vida familiar; participación comunitaria y política; oportunidades de educación, desarrollo personal y trabajo; vivienda adecuada; acceso a una justicia expedita; y estar libre de discriminación, violencia y abuso. Aunque la mayoría de estos derechos son evidentemente derechos de todas las personas dentro de la jurisdicción del Estado, sostenemos que son susceptibles de un significado específico en el contexto de las personas mayores. La tendencia a constitucionalizar los derechos de las personas mayores demuestra un apoyo creciente a la idea de que las personas mayores son una categoría de humanidad cuyas necesidades y experiencias son lo suficientemente irreductibles como para merecer un reconocimiento especial en la ley. ${ }^{240}$

El capítulo procede de la siguiente manera: la primera sección cubre los instrumentos internacionales que abordan explícitamente los derechos de las personas mayores, contemplando diferentes regiones por separado para resaltar algunas de sus diferencias en el abordaje del tema. Dadas las lagunas a nivel internacional y regional, la constitucionalización de los derechos de las personas mayores a menudo ha asumido un papel destacado y renovado, siendo luego examinada en la segunda parte desde una perspectiva más sintética y transversal para destacar las principales evoluciones de los derechos. 
Si bien no existe un solo tratado de la ONU dedicado a los derechos de las personas mayores, existe un mosaico de disposiciones de tratados de la ONU que reconocen derechos de relevancia específica para las personas mayores. Esta sección también identifica las protecciones progresistas en los tratados regionales africanos e interamericanos sobre los derechos de las personas mayores, al tiempo que reconoce que el tratado africano carece de la membresía necesaria para entrar en vigor, mientras que el tratado interamericano carece de la membresía necesaria para establecer mecanismos formales para monitorear su implementación.

\section{Sistema de derechos humanos de la ONU}

El Pacto Internacional de Derechos Civiles y Políticos (PIDCP) y el Pacto Internacional de Derechos Económicos, Sociales y Culturales (PIDESC) ofrecen protecciones no específicas a las personas mayores.

Los derechos en el Pacto Internacional de Derechos Civiles y Políticos de interés especial para las personas mayores son la libertad de conciencia, expresión, reunión y asociación, incluido el no proselitismo (arts. 18-19, 21); el derecho a elegir la propia residencia (art. 12) y a no sufrir injerencias arbitrarias en el hogar, la intimidad o la familia (art. 17 (1)); el derecho a participar en la vida política y cívica (art. 25 a) y b)); y a acceder a los servicios públicos en igualdad de condiciones con los demás (art. 25 c)).

Los derechos de especial interés en el PIDESC incluyen condiciones de trabajo seguras y apropiadas (art. 7 (b)); el derecho a la seguridad social (art. 9) y a un nivel de vida adecuado (art. 11 (1)); el derecho a disfrutar del más alto nivel posible de salud física y mental (art. 12); y el derecho a participar en la vida cultural (art. 15).

Otros tratados de la ONU que abordan específicamente los derechos de los grupos desfavorecidos, proporcionan algunas protecciones específicas por edad. El art. 11 (1) (e) de la Convención sobre la Eliminación de Todas las Formas de Discriminación contra la Mujer (CEDAW) especifica un derecho igualitario de mujeres y hombres a la seguridad social, "en particular en casos de jubilación, desempleo, enfermedad, invalidez (...) [y] vejez". La Convención sobre la protección de los derechos de los trabajadores migratorios y de sus familiares (CMW) incluye la "edad" en el art. 7, dentro de los motivos prohibidos de discriminación en relación con los derechos establecidos en el tratado, aunque no se limita a la vejez.

Si bien no todas las personas mayores son personas con discapacidad (PCD), la Convención sobre los derechos de las personas con discapacidad
B.

\section{El marco}

internacional 
(CDPD) incluye protecciones que cubren la intersección de la edad y la discapacidad. Estos son, el art. 8 que obliga a los Estados miembros a abordar los estereotipos y prejuicios relacionados con las personas con discapacidad, incluidos los basados en el sexo y la edad; el art. 9 sobre igualdad de acceso al entorno construido, la información y los servicios públicos; el art. 13(1) sobre "ajustes de procedimiento (...) adecuados a la edad" para facilitar el papel de las personas con discapacidad de edad avanzada como participantes directos e indirectos en el sistema de justicia; y los arts. 19, 20 y 26 sobre medidas apropiadas para permitir la vida independiente, la movilidad personal y la (re)habilitación para maximizar la independencia y la participación en todos los aspectos de la vida.

\section{Sistemas regionales de los derechos humanos}

África y las Américas son las únicas dos regiones con instrumentos jurídicamente vinculantes dedicados a los derechos de las personas mayores.

- África

La Carta Africana de Derechos Humanos y Derechos de los Pueblos de 1981 es el primer instrumento de derechos humanos del mundo que reconoce y protege expresamente los derechos de las personas mayores como una categoría distinta de derechos. ${ }^{241}$ El art. 18(4) de la Carta Africana establece que "Los ancianos y los minusválidos tendrán derecho a medidas especiales de protección adecuadas a sus necesidades físicas o morales". A pesar de su tratamiento distintivo de los derechos de las personas mayores, el art. 18(4) ha recibido críticas por agrupar a mujeres, niños y personas con discapacidad con personas mayores, como si sus necesidades y experiencias fueran las mismas o en gran medida similares. ${ }^{242}$

En 2002, la Unión Africana (UA) aprobó el Marco Africano de Política Pública y Plan de Acción sobre el Envejecimiento, que pedía específicamente la adopción de un protocolo adicional a la Carta Africana relacionado con los derechos de las personas mayores. ${ }^{243}$ La UA adoptó el Protocolo de la Carta Africana sobre los Derechos de las Personas Mayores en 2016.

Curiosamente, el Protocolo no detalla expresamente los derechos de las personas mayores, sino que los articula indirectamente al definir las obligaciones de los Estados Partes. Obliga a las partes a eliminar la discriminación contra las personas mayores (art. 3); y proporcionar un acceso equitativo al

\footnotetext{
241 Chirway Rushwaya, 2019, p. 60.

242 Ibíd.

243 Ibíd., p. 54, citando la recomendación del Marco Africano de Política Pública y Plan de Acción sobre el Envejecimiento, 1(a) del párrafo 6.1.
} 
empleo (art. 6), la seguridad social (art. 7), los servicios de salud (art. 15), la educación (art. 16) y la infraestructura (art. 18). Este enfoque, basado en las obligaciones del Estado en lugar de centrarse en los derechos per se, no es desconocido particularmente en el contexto de la discapacidad, y puede ser apropiado cuando el Estado requiere importantes inversiones en bienestar.

Existen disposiciones específicas que protegen a las mujeres mayores de la violencia de género (art. 9) y de prácticas tradicionales nocivas como las acusaciones de brujería (art. 8); proporcionar a las personas mayores rescate de emergencia, asentamiento y repatriación, con carácter prioritario (art. 14); y proveen apoyo financiero a las personas mayores indigentes que se ocupan de los niños huérfanos por la epidemia del SIDA/VIH (art. 12).

Además, la inclusión en el Protocolo del deber de las personas de edad de orientar y transmitir conocimientos y experiencias a las generaciones más jóvenes y de fomentar y facilitar el diálogo y la solidaridad intergeneracionales (art. 20) es particularmente singular y progresista.

Sin embargo, al 15 de enero de 2021, sólo dos países habían ratificado el Protocolo (Lesotho y Benin) y se necesitan quince ratificaciones para que entre en vigor. ${ }^{244}$

\section{- Las Américas}

La Convención Interamericana sobre los Derechos de las Personas Mayores (CIDPM) es el primer tratado internacional dedicado a la protección de los derechos de las personas mayores, adoptado en 2015 y vigente desde 2017. Ciertas disposiciones son particularmente progresistas y hablan directamente a los obstáculos ambientalmente específicos que enfrentan las personas mayores para garantizar sus derechos. El art. 12, por ejemplo, garantiza que se respeten todos los derechos humanos en los entornos de cuidados de larga duración, subrayando el acceso a la información (art. 12 (i)); la protección de su integridad, privacidad e intimidad (art. 12 (c) (v)); y el derecho a la recreación, el esparcimiento y el deporte (art. 22) para mejorar su salud y calidad de vida y promover su autonomía y realización personal. El art. 24 especifica el derecho a una vivienda que sea segura y que pueda adaptarse a las necesidades de las personas mayores.

244 Listado de países que han firmado, ratificado o accedido al Protocolo a la Carta Africana de derechos Humanos y de los Pueblos sobre los derechos de las personas mayores: https://au.int/sites/default/files/treaties/36438-sl-PROTOCOL\%20TO\%20THE\%2OAFRICAN\%20CHARTER\%200N\%20HUMAN\%20AND\%20 PEOPLES\%E2\%80\%99\%20RIGHTS\%200N\%20THE\%20RIGHTS\%200F\%20 OLDER\%2OPERSONS.pdf. 
Al 15 de enero de 2020, solo 7 países habían ratificado la Convención. Esto significa que el tratado tiene tres miembros menos de los diez necesarios para establecer los mecanismos de seguimiento establecidos en el tratado para monitorear su implementación.

- Europa

Hasta que la Carta de los Derechos Fundamentales de la Unión Europea entró en vigor en 2009, los derechos de las personas mayores no estaban expresamente reconocidos en la legislación de la UE. ${ }^{245}$ Incluso hoy en día, sólo se encuentran algunas referencias dispersas a nivel regional en Europa.

El art. 21 de la Carta brinda protección explícita contra la discriminación por edad. El art. 25 especifica que "[l]a Unión reconoce y respeta el derecho de las personas mayores a llevar una vida digna e independiente y a participar en la vida social y cultural". El art. 25 se dice que marca un cambio desde un enfoque bienestarista tradicional de las necesidades de las personas mayores hacia uno basado en los derechos y fundado en el empoderamiento y la participación de las personas mayores. ${ }^{246} \mathrm{El}$ art. 34 reconoce el derecho de las personas mayores a una red de protección social no restringida a las personas en el entorno laboral. ${ }^{247}$ Sin embargo, esta canasta de derechos para las personas mayores no es equivalente -en términos de la ambición- con los marcos comprensivos de derechos en el nivel regional de África y las Américas.

\section{El marco doméstico}

En ocasiones, las constituciones nacionales pueden ir más allá de lo que exigen estrictamente los instrumentos internacionales de derechos humanos, o al menos enmarcar la protección de los derechos de las personas mayores de manera diferente, y en formas que se erigen para enriquecer el registro de enfoques disponibles. Si bien los derechos que identificamos en las disposiciones constitucionales no son derechos inherentes a las personas mayores, estas disposiciones reenfocan, reformulan o adaptan de manera importante los derechos existentes para cubrir mejor las necesidades y experiencias propias de las personas mayores. Además, clasificar estas disposiciones ayuda a comprender mejor cómo pueden afectar la ontología de los derechos, ya que algunas disposiciones formulan derechos para las personas mayores que nunca se han enmarcado como tales en los instrumentos de derechos humanos. 
Estas disposiciones constitucionales pueden considerarse pertenecientes a una de tres categorías. Primero, hay disposiciones que aclaran cómo se aplican los derechos existentes a las personas mayores. Éstas pueden estar reafirmando lo obvio, pero ciertamente ayudan a asegurar que las personas mayores no sean olvidadas como igualmente dignas de la protección de esos derechos. En segundo lugar, están las disposiciones que reformulan, amplían o adaptan el contenido de los derechos existentes para cubrir mejor las necesidades y experiencias inherentes a las personas mayores. Tal movimiento no crea necesariamente nuevas categorías de derechos porque se relacionan con derechos existentes, pero agrega alcance y complejidad a los elementos que podrían requerirse para realizar esos derechos. Finalmente, dejamos al descubierto disposiciones que detallan deberes en lugar de derechos. Las disposiciones del tercer tipo pueden atribuir, a través de la constitucionalización, importancia moral a un deber del Estado sin depender del "discurso" de derechos, lo que conlleva un bagaje conceptual considerable. Sin embargo, para todos los efectos y propósitos, ellos pueden ser vistos como "la otra cara" de los derechos.

\section{Disposiciones que aclaran cómo los derechos existentes se aplican a las personas mayores}

Las disposiciones constitucionales que aclaran cómo se aplican los derechos existentes a las personas mayores hacen que las personas mayores se incluyan de manera más evidente dentro de estos derechos en un contexto donde la discriminación por edad podría no hacer que parezcan beneficiarios obvios. Esto puede ayudar a ver cómo las violaciones de estos derechos afectan a las personas mayores de manera particular y anticipar las barreras edad-específicas para el disfrute de estos derechos.

2. No discriminación, igualdad y el derecho al empleo (arts. 3, 4 y 26 del PIDCP; art. 6 del PIDESC; y arts. 5 y 18 del CIDPM)

Las constituciones de Venezuela de 1999 (art. 89 (5)) y Paraguay de 1992 (art. 88) abordan la realidad de que las personas pueden necesitar seguir trabajando en la vejez y proporcionan protecciones para prohibir específicamente la discriminación basada en la edad en el lugar de trabajo. Estos artículos parecen centrarse en el lugar de trabajo, en particular, como un lugar de discriminación para las personas mayores. El art. 88 de la Constitución de Paraguay especifica además que el "trabajo de las personas con limitaciones o incapacidades físicas o mentales será especialmente amparado". Esta disposición identifica una razón clave por la cual las personas mayores pueden ser discriminadas en el lugar de trabajo, anticipando cómo las personas mayores pueden ser marginadas por la industrialización. 
Más allá de la cuestión de la discriminación, la Constitución de Irlanda de 1939 garantiza un trabajo adecuado a la edad. El art. 45(2) de la Constitución, garantiza que los ciudadanos irlandeses no se vean obligados por necesidades económicas a realizar un trabajo inadecuado para su edad y fuerza. Si entendemos la igualdad no como un trato equivalente, sino como un trato sensible a las necesidades que permiten a las personas vivir en igualdad de condiciones, entonces el trabajo apropiado para la edad puede verse como una medida que brinda a las personas mayores oportunidades -sensibles a sus necesidades- para trabajar en igualdad de condiciones con los demás.

Las edades de jubilación obligatorias se consideran un trato desigual injustificado y, por lo tanto, una discriminación, ya que exigen la jubilación en función de la edad y no de un factor justificado, como la capacidad de una persona para realizar su función laboral. Tanto la jubilación obligatoria como su rechazo constitucional por parte de las personas mayores ha sido causa de polémica. El fundamento tradicional de la política de jubilación obligatoria es brindar a las generaciones más jóvenes la oportunidad de participar en la economía y ganarse la vida. Enmarcado de esta manera, la jubilación obligatoria podría considerarse como una intervención afirmativa para promover la solidaridad intergeneracional, un principio que se entreteje a través del CIDPM y el Protocolo de la UA. ${ }^{248}$

Sin embargo, el aumento de los costos de vida, el estancamiento de los salarios y la erosión del estado de bienestar en muchas partes del mundo significan que una proporción creciente de la población puede verse obligada a trabajar después de la edad de jubilación por razones financieras. En la actualidad, en Europa, hay una promedio de 3,4 personas en edad laboral para apoyar la jubilación de las personas de 65 años o más. Para 2050, se prevé que ese número disminuya a 2. ${ }^{249}$ En los EE.UU., cerca del $20 \%$ de las personas de 45 a 59 años informan una falta total de ahorros para la jubilación y esa cifra es del $13 \%$ para las personas de 60 años o más. ${ }^{250}$ Estas tendencias reflejan un problema global. En la mayoría de los países, el riesgo de pobreza aumenta con la edad. ${ }^{251}$ Obligar a las personas a dejar de trabajar en un entorno en el que no se pueden garantizar unos ingresos mínimos puede, por tanto, afectar no sólo su derecho al trabajo, sino también su derecho a la salud o incluso su derecho a la vida. Un mejor enfoque

\footnotetext{
248 Convención Interamericana sobre los Derechos de las Personas Mayores, art. 3(j); Protocolo de la Carta Africana sobre los Derechos de las Personas Mayores, art. 20(2).

249 Amaglobeli, Dabla-Norris y Gaspar, 2020, p. 31.

250 Board of Governors of the Federal Reserve System, 2019, pp. 47-48.

251 Department of Economic and Social Affairss programe on ageing, sin fecha, p. 1.
} 
es aquel que simultáneamente no hace obligatoria la jubilación y ayuda a quienes buscan jubilarse más allá de cierta edad a hacerlo, salvaguardando así las dimensiones negativas y positivas del derecho al trabajo.

\section{Derecho a una vivienda adecuada (art. 11 del PIDESC; art. 24 del CIDPM)}

El derecho a la vivienda permite el disfrute de otros derechos y condiciones necesarios para una vida digna. ${ }^{252}$ Una vivienda adecuada puede permitir el derecho al trabajo. Pone a sus beneficiarios cerca de las opciones de empleo. A medida que nuestros hogares se han convertido en nuestros espacios de trabajo durante la pandemia de COVID-19, un entorno hogareño seguro, higiénico, bien iluminado y bien ventilado es esencial para el trabajo y la empleabilidad. Además, a menudo se requiere una dirección permanente para buscar trabajo y abrir una cuenta bancaria. Una vivienda adecuada también promueve la salud al proteger a las personas de los peligros ambientales y los vectores de enfermedades, proporcionar agua potable y ponerlos al alcance de los servicios de salud. El art. 24 de la CIDPM reconoce que tener un hogar puede facilitar la asignación de atención médica, en particular de atención continua.

Las constituciones de Angola de 2010 (art. 82), Portugal de 1976 (art. 72) y Mozambique de 2004 (art. 124), incluyen específicamente el derecho a la vivienda de las personas mayores y reconocen expresamente este derecho como vital para su autonomía y para prevenir el aislamiento y marginación social. Un hogar adecuado es un espacio digno que puede facilitar una vida familiar y comunitaria y es una condición previa para la privacidad y la intimidad con los demás. Esto es particularmente clave para las personas mayores alojadas en centros de atención de larga estadía, ya que vivir en este tipo de entorno significa ser vulnerable a diversos grados de paternalismo y puede implicar la negociación, y a veces la ruptura, de la distinción público-privado.

\section{Participación en la gobernanza y en el proceso político (arts. 19, 21, 22 y 25 del PIDCP; arts. 27 y 28 del CIDPM)}

Como categoría de la humanidad, las personas mayores ejemplifican la paradoja poder/vulnerabilidad. ${ }^{253}$ En general, las personas mayores tienen un poder y una influencia políticos considerables y un capital financiero. Predominan en los puestos más altos del poder, desde la junta directiva de las empresas Fortune 500 hasta los principales puestos de formulación 
de políticas públicas. Las personas mayores pueden ser más ricas en promedio, concentrar una parte relativamente alta del capital y, al menos en Occidente, se considera que tienen amplias oportunidades de recreación y esparcimiento durante la jubilación. ${ }^{254}$

Al mismo tiempo, envejecer puede significar vivir fuera de la vista, y con una libertad disminuida, en situaciones de vulnerabilidad inherente. Esto es particularmente cierto en el caso de las personas mayores bajo tutela legal o admitidas en un centro de atención de larga estadía. A menudo, en estos escenarios, se presume que las personas mayores carecen de capacidad jurídica y pueden estar en una relación de subordinación mediada por el Estado que obstaculiza en gran medida su capacidad para conducir su propia vida o defender sus intereses. Esto tiene efectos obvios y graves en la capacidad de una persona mayor para asociarse y reunirse con otros para dejar su huella en la vida pública y política.

La Constitución de Tailandia de 2017 incluye una disposición con visión de futuro que promueve la representación de los intereses de las personas mayores en la legislatura nacional. El art. 128 requiere que los comités ad hoc para la legislación relativa a las personas mayores estén compuestos en un tercio por personas mayores o representantes de ONG directamente interesadas en los derechos de las personas mayores. Dada la preocupación por la falta de capacidad jurídica y libertad, esta disposición es un complemento importante de otras mecanismos formales de participación política, como el derecho al voto, que requiere capacidad jurídica.

\section{Acceso a una justicia expeditiva y apropiada a la edad (art. 14(c) del PIDCP; art. 13(1) del PIDESC; art. 31 del CIDPM)}

El hecho de que las personas mayores tengan menos vida por delante se ha considerado relevante para evaluar si los procedimientos se han retrasado injustificadamente. ${ }^{255}$ Otro factor clave para una justicia expedita es el tipo específico de problemas que los adultos mayores llevan ante los tribunales, algunos de los cuales son particularmente urgentes y pueden ameritar un tratamiento acelerado. Las disputas de tutelas y guardas deben tratarse rápidamente porque generalmente implican la pérdida severa de la autonomía personal y la propiedad, entre otras libertades. Las disputas sobre pensiones merecen una determinación rápida cuando se depende de una pensión para salir adelante.

\footnotetext{
254 Ibíd.

255 Jablonska v Poland, 2004; Sussman v Germany, 1996.
} 
El acceso a una justicia expedita también es vital en los casos de violencia o abuso doméstico que involucran a personas mayores donde una víctima mayor se encuentra en una relación de dependencia con su agresor. El art. 81 de la Constitución del Ecuador reconoce este hecho social y obliga al Estado a proporcionar fiscales y defensores especiales y procedimientos expeditos para conocer de los casos de violencia intrafamiliar y otros delitos perpetrados contra las personas mayores. Estas medidas reconocen que el sistema legal generalmente asume un cierto grado de habilidad, comprensión de la ley y el sistema legal e independencia para tomar decisiones con implicaciones de por vida, por parte de los participantes; supuestos que no siempre son precisos para la población promedio y es probable que lo sea aún menos que el promedio. Este es particularmente el caso cuando, en el contexto de la justicia penal, la revictimización del trauma y la vergüenza puede estar involucrada.

\section{Derecho a la familia y a participar en la vida comunitaria (Art 23(1) del PIDCP; arts. 19 y 29 de la CDPD; art. 3(j) t 8 del CIDPM)}

El Plan de Acción Internacional de Madrid sobre el Envejecimiento (PAIME) de 2002 puso de manifiesto cómo las personas mayores de todo el mundo corren un mayor riesgo de soledad y marginación social. Es más probable que enfrenten la pérdida de sus seres queridos, e impedimentos como la pérdida de audición y la movilidad reducida, en combinación con obstáculos ambientales y barreras sociales y de actitud, hacen que sea relativamente más difícil para ellos mantener vínculos sociales significativos y participar en la vida comunitaria. ${ }^{256}$

La forma que adoptan estos problemas varía en función de si las personas mayores viven en entornos urbanos o rurales, o están pasando de un entorno rural a otro urbano. En las economías en transición, la marcha de las generaciones más jóvenes a las ciudades en busca de oportunidades laborales significa que las personas mayores pueden quedarse atrás en las comunidades rurales sin el apoyo familia. ${ }^{257}$ Además, las personas de edad avanzada en los países en desarrollo que hacen la transición de zonas rurales a las urbanas, a menudo se enfrentan a la pérdida de sus redes sociales y adolecen de una falta de infraestructura y servicios accesibles en las ciudades, lo que puede conducir a su marginación y exclusión. ${ }^{258}$

\footnotetext{
256 Declaración Política y Plan de Acción Internacional de Madrid sobre el Envejecimiento, 2002, pár. 29.

257 Ibíd.

258 Ibíd., pár. 31.
} 
El PAIME establece conexiones importantes entre la calidad del entorno de vida de una persona mayor, la vida familiar y su riesgo de aislamiento y marginación social. Sin embargo, la conexión entre estos factores también se aborda en las constituciones de Angola de 2010 (art. 82), Portugal de 1976 (art. 72) y Mozambique de 2004 (art. 124), que tratan la vivienda, la familia y la vida comunitaria como elementos integrales que "eviten y superen su aislamiento o su marginación social". ${ }^{259}$ La vivienda y la situación de vida de una persona mayor pueden tener un impacto considerable en su capacidad para establecer conexiones sociales, moverse libremente e incluso determinar si hay una comunidad a su alrededor en la que participar. Además, el rol de la familia en la prevención o lucha contra el aislamiento y la marginación de las personas mayores se enfatiza aún más en las constituciones de Brasil (arts. 230), Colombia de 1991 (art. 46) y República Dominicana (art. 57), que especifican el papel activo que debe desempeñar la familia en estos asuntos. Por ejemplo, el art. 46 de la constitución colombiana exige que la familia, con respecto a las personas mayores, promueva "su integración a la vida activa y comunitaria".

Estas disposiciones proporcionan una perspectiva específica para las personas mayores sobre el derecho a la familia del art. 23(1) del PIDCP. El Pacto Internacional de Derechos Civiles y Políticos se centra en general en la primacía y la integridad de la familia y el papel del Estado y la sociedad en su protección y promoción. Las disposiciones constitucionales analizadas, en cambio, abordan los deberes familiares hacia los miembros mayores de la familia, incluido el deber de integrar socialmente a las personas mayores, a medida que pierden sus lazos sociales significativos al envejecer y requieren apoyo para crear otros nuevos.

\section{Protección contra la violencia y el abuso (art. 16 de la CDPD; art. 9 del CIDPM)}

Las personas mayores son particularmente susceptibles a la violencia y el maltrato. Una reducción en la capacidad y la fuerza física puede ponerlos en relaciones de dependencia y en mayor riesgo de violencia y abuso. La violencia y el abuso también pueden perpetuarse en su contra sobre la base de estereotipos negativos y prejuicios que se convierten en prácticas sociales nocivas, como las acusaciones de brujería contra mujeres mayores en África. ${ }^{260}$ Se podría suponer que la libertad frente a la violencia y el abuso es un derecho de larga data, pero de hecho se codificó por primera vez en el art. 16 de la CDPD en términos de la protección "contra todas las formas de explotación, violencia y abuso". ${ }^{261}$ 
La Constitución de Bolivia de 2009 especifica una prohibición clara contra toda forma de maltrato, violencia y abandono contra las personas mayores (art. 68 (ii)). Curiosamente, "abandono" se agrupa con "maltrato" y "violencia" en esta disposición. El abandono de personas adultas mayores por "familiares o las instituciones establecidas para su protección" también es punible por la ley en virtud del art. 38 de la Constitución de Ecuador de 2008. Dicho esto, se puede argumentar que el "derecho a no ser abandonado" no es un derecho exigible. Es difícil determinar cuándo el umbral del "abandono" se alcanza, cuándo el deber de no abandonar se compromete y qué actores son responsables de cumplir con ese deber.

Puede que no exista el derecho a no ser abandonado, pero alguna situación particular puede imponer el deber moral de no abandonar a otros. Al igual que las acciones, las inacciones y las omisiones pueden conducir a resultados moralmente repugnantes. ${ }^{262}$ En situaciones en las que una persona mayor depende en gran medida de otra para recibir apoyo vital y esa dependencia da lugar a una relación de dependencia, el abandono puede ser moralmente equivalente a un maltrato activo. Las disposiciones anteriores en las constituciones de Bolivia y Ecuador y en la CIDPM parecen reconocer esta realidad social.

Esta subsección identifica las disposiciones constitucionales que reformulan o amplían los derechos existentes. Si bien estas disposiciones no transmiten derechos inherentes a las personas mayores per se, hablan de la necesidad de introducir leyes que prioricen ciertos aspectos de la experiencia humana inherentes a las personas mayores.

\section{El derecho a la asistencia médica especializada y medicamentos gratuitos}

A medida que nuestra población envejece, las sociedades deben tomar decisiones difíciles con respecto a la distribución de los escasos recursos sanitarios. Una de las principales preocupaciones son los costos de los medicamentos de mantenimiento y los tratamientos especializados para tratar los problemas de salud asociados con el envejecimiento y cuyo objetivo es permitir que las personas mayores tengan una vida más larga y saludable.

El derecho a la salud se establece en términos generales en las constituciones de Egipto de 2014 (art. 83) y Gabón de 1991 (art. 1 (8)), y ha sido criticado por ser vago e incapaz de proporcionar mucha orientación sustantiva
D.

Reformulación o expansión de los derechos existentes

262 Jarvis Thomson, 1985, pp. 1395-1415. 
sobre cómo distribuir los recursos de la salud. ${ }^{263}$ El art. $37(1)$ de la Constitución de Ecuador establece prioridades específicas y brinda orientación sobre dicha distribución de recursos. Estipula el derecho a una atención médica especializada gratuita, así como el acceso gratuito a los medicamentos. Esto podría verse como una adaptación del derecho a la salud para los propósitos de las personas mayores que aborda un punto que de otra manera no estaría claro. El art. 19(m) de la CIDPM indica que los medicamentos reconocidos como esenciales por la Organización Mundial de la Salud deben estar disponibles y accesibles para las personas mayores. Se podría decir que el art. 37(1) de la Constitución de Ecuador va más allá al detallar qué se entenderá por accesible (gratuito).

\section{Derecho a un salario mínimo para personas indigentes}

El art. 203(v) de la Constitución brasileña de 1988 garantiza expresamente un salario mínimo a las personas mayores indigentes, independientemente de si han cotizado en el sistema de seguridad social. La mayoría de los trabajadores de la economía informal no tienen acceso a la protección social, incluida la seguridad social. Los datos de la Encuesta Nacional de Hogares a principios de 2020 muestran que alrededor de 38 millones de personas en Brasil trabajaban en la economía informal. ${ }^{264} \mathrm{El}$ art. 203(v) podría considerarse una ampliación del derecho a la seguridad social que se encuentra en el art. 9 PIDESC y en el art. 17 de la CIDPM para cubrir a los trabajadores excluidos de la seguridad social cuando llegan a la vejez.

E.

Disposiciones que crean deberes estatales en vez de un derecho

Es perfectamente coherente referirse a las obligaciones del Estado sin referirse a los derechos correspondientes. No todos los deberes estatales de gran importancia moral deben formularse como un derecho. Hablar de los deberes del Estado sin invocar derechos puede ayudar a eludir los problemas de indeterminación de algunos supuestos derechos al tiempo que se atribuye importancia moral a un deber del Estado.

El art. 38 de la Constitución del Ecuador especifica un marco de obligaciones para el cumplimiento de derechos específicos de las personas mayores adelantados en el art. 37 (por ejemplo, derecho al trabajo remunerado, jubilación universal, etc.). Los marcos de obligaciones amplios proporcionan una buena señal de lo que generalmente se requiere para implementar el derecho en cuestión. Por ejemplo, para implementar el derecho de las personas mayores al trabajo remunerado (art. 37 (2)), el Estado está obligado

\footnotetext{
263 Daniels, 2008, capítulo 12.

264 Nogueira, Amaral y Jones, 2020.
} 
a fomentar la participación de las personas mayores en las instituciones públicas y privadas y debe desarrollar programas de capacitación laboral, basados en su profesión y ambiciones a tal efecto (art. 38 (2)).

El ámbito de la protección de los derechos de las personas mayores está cambiando y es relativamente nuevo. Como hemos señalado, hay una serie de iniciativas internacionales influyentes, sobre todo regionales, que han tratado de orientar esos esfuerzos. Pero las prácticas constitucionales, algunas impulsadas por la implementación internacional y otras en gran parte de cosecha propia, también evidencian un rico registro de normas que apuntan a captar mejor la especificidad de la experiencia de los derechos de las personas mayores. Esto debería crear auténticas aperturas para la experimentación constitucional que reconozca la contribución y los límites de lo que el discurso de los derechos puede hacer por las personas mayores. En algunos casos, todo lo que se necesitará es la protección general e igual de los derechos de todos; en otros casos, puede ser útil explicar con más precisión qué significa un derecho general específicamente para las personas mayores. Luego, esto puede enmarcarse como parte de debates sociales más amplios sobre la solidaridad intergeneracional o la dignidad en la vejez. El punto fundamental sigue siendo que las vulnerabilidades específicas de las personas mayores deben tomarse en serio en cualquier contexto constitucional. 
Afganistán, Algeria, Angola, Argentina, Australia, Baréin, Bangladés, Bélgica, Benín, Bolivia, Brasil, Bulgaria, Burkina Faso, Camboya, Camerún, Canadá, Cabo Verde, República Centroafricana, Chad, China, Colombia, Congo, Costa Rica, Côte d'Ivoire, Croacia, Cuba, República Checa, República Dominicana, Ecuador, Egipto, El Salvador, Etiopía, Eritrea, Estonia, Finlandia, Gabón, Gambia, Ghana, Guatemala, Guinea, Guinea-Bissau, Guyana, Haití, Honduras, Hungría, India, Irán, Iraq, Irlanda, Italia, Jordania, Kenia, Kuwait, Kyrgyzstan, Laos, Latvia, Libia, Lituania, Madagascar, Malaui, Malasia, Maldivas, Malta México, Mongolia, Marruecos, Mozambique, Myanmar, Nicaragua, Nepal, Omán, Panamá, Paraguay, Perú, Polonia, Portugal, Qatar, Rusia, Ruanda, Senegal, Seychelles, Sierra Leona, Eslovaquia, Sudáfrica, España, Somalia, Corea del Sur, Sudán del Sur, Sudan, Suazilandia, Suecia, Siria, Tayikistán, Timor-Leste, Tailandia, Togo, Turkmenistán, Uganda, Emiratos Árabes Unidos, Uruguay, Uzbekistán, Venezuela, Vietnam, Sahara Occidental, Yemen y Zimbabue.

Angola, Argentina, Bolivia, Brasil, Canadá, Colombia, Costa Rica, Cuba, República Dominicana, Ecuador, Egipto, El Salvador, Estonia, Finlandia, Gabón, Guatemala, Honduras, Irlanda, Kenia, Malaui, Perú, Portugal, Sudáfrica, España, Suecia, Uruguay, Venezuela, Tailandia y Uganda. 
Amaglobeli, D., Dabla-Norris, E. y Gaspar, V. (2020)

'Getting Older but Not Poorer', Finance \& Development, 57(1). Disponible en: https://www.imf.org/external/pubs/ft/fandd/2020/03/impact-of-aging-onpensions-and-public-policy-gaspar.htm (Consulta: 24 agosto 2021).

Board of Governors of the Federal Reserve System (2019)

Report on the Economic Well-Being of U.S. Households in 2018. Disponible en: https://www.federalreserve.gov/publications/files/2018-report-economic-well-being-us-households-201905.pdf (Consulta: 25 noviembre 2020).

Carta Africana de Derechos Humanos y de los Pueblos (1981) Disponible en: https://www.acnur.org/fileadmin/Documentos/ BDL/2002/1297.pdf (Consulta: 24 agosto 2021).

Carta de los Derechos Fundamentales de la Unión Europea (2009)

Disponible en: https://eur-lex.europa.eu/legal-content/EN/TXT/?uri=CELEX:12012P/TXT (Consulta: 10 diciembre 2020).

Chirwa, D. y Rushwaya, C. (2019)

'Guarding the Guardians: A Critical Appraisal of the Protocol to the African Charter on the Rights of Older Persons in Africa, Human Rights Law Review, 19(1). DOI: 10.1093/hrlr/ngy046.

Comité de Derechos Económicos, Sociales y Culturales (1991)

Observación general $N^{\circ} 4$. El derecho a una vivienda adecuada (párrafo 1 del artículo 11 del Pacto). Disponible en: https://tbinternet.ohchr.org/_layouts/15/treatybodyexternal/Download.aspx?symbolno=INT\%2fCESCR\%2fGEC\%2f4759\&Lang=en (Consulta: 20 noviembre 2020).

Convención Americana sobre Derechos Humanos (1969)

Disponible en: https://www.oas.org/dil/esp/tratados_b-32_convencion_ americana_sobre_derechos_humanos.htm (Consulta: 24 agosto 2021).

Convención Interamericana sobre la protección de los derechos humanos de las personas mayores (2015)

Disponible en: http://www.oas.org/es/sla/ddi/tratados_multilaterales_interamericanos_a-70_derechos_humanos_personas_mayores.asp (Consulta: 24 agosto 2021).

Convención internacional sobre la protección de los derechos de todos los trabajadores migratorios y de sus familiares (1990)

Disponible en: https://www.ohchr.org/sp/professionalinterest/pages/cmw. aspx (Consulta: 24 agosto 2021). 
Convención Internacional sobre los Derechos de las Personas con Discapacidad (2016)

Disponible en: https://www.un.org/esa/socdev/enable/documents/tccconvs. pdf (Consulta: 24 agosto 2021).

Convención sobre la Eliminación de Todas las Formas de Discriminación Contra la Mujer (1988)

Disponible en: https://www.ohchr.org/sp/professionalinterest/pages/cedaw. aspx (Consulta: 24 agosto 2021).

Convenio Europeo de Derechos Humanos (1950)

Disponible en: https://www.echr.coe.int/Documents/Convention_SPA.pdf (Consulta: 24 agosto 2021).

Daniels, N. (2008)

Just Health: Meeting Health Needs Fairly. Cambridge UK: Cambridge University Press.

Declaración Política y Plan de Acción Internacional de Madrid sobre el Envejecimiento (2002)

Segunda Asamblea Mundial sobre el Envejecimiento.

Disponible en: https://social.un.org/ageing-working-group/documents/mipaa-sp.pdf (Consulta: 24 agosto 2021).

Department of Economic and Social Affairs programme on ageing (sin fecha) Income Poverty in Old Age: An Emerging Development Priority. Disponible en: https://www.un.org/esa/socdev/ageing/documents/PovertylssuePaperAgeing.pdf (Consulta: 24 agosto 2021).

Doron, I. (2013)

'Older Europeans and the European Court of Justice', Age and Ageing, 42(5). DOI: 10.1093/ageing/aft053.

Doron, I. et al. (2018)

'Ageism and Anti-Ageism in the Legal System: A Review of Key Themes', en AyaIon, L. y Tesch-Römer, C. (eds.) Contemporary Perspectives on Ageing. Springer Cham, pp. 303-319. DOl: 10.1007/978-3-319-73820-8_19.

European Union Agency for Fundamental Rights (2018)

Fundamental Rights Report 2018. Disponible en: https://fra.europa.eu/en/publication/2018/fundamental-rights-report-2018 (Consulta: 24 agosto 2021). 
Fredvang, M. y Biggs, S. (2012)

'The Rights of Older Persons: Protection and gaps under human rights law', Social Policy Working Paper, 16. Disponible en: https://social.un.org/ageing-working-group/documents/fourth/Rightsofolderpersons.pdf (Consulta: 24 agosto 2021).

Hassoun, N. (2019)

'The Human Right to Health: A Defense', Journal of Social Philosophy, 51(2):158-179. DOl: 10.1111/josp.12298.

Jablonska v Poland (2004)

Corte Europea de Derechos Humanos. Caso No. 60225/00.

Mégret, F. (2008)

'The Disabilities Convention: Human Rights of Persons with Disabilities or Disability Rights?', Human Rights Quarterly, 30(2):494-516.DOI: 10.1353/ hrq.0.0000.

Mégret, F. (2011)

'The Human Rights of Older Persons', Human Rights Law Review, 11(1). DOI: 10.1093/hrlr/ngq050.

Müller, C. y Sanderson, S. (2020)

'Witch hunts: A global problem in the 21st century', Deutsche Welle, 10 agosto. Disponible en: https://www.dw.com/en/witch-hunts-a-global-problem-inthe-21st-century/a-54495289 (Consulta: 24 agosto 2021).

Nogueira, M., Amaral, A.I. y Jones, G. (2020)

'The impact of COVID-19 on Brazil's precarious labour market calls for far-reaching policies like universal basic income,' LSE Blog, 3 junio. Disponible en: https://blogs.Ise.ac.uk/latamcaribbean/2020/06/03/the-impact-of-covid-19on-brazils-precarious-labour-market-calls-for-far-reaching-policies-like-universal-basic-income/ (Consulta: 24 agosto 2021).

Pacto Internacional de Derechos Civiles y Políticos (1966)

Disponible en: https://www.ohchr.org/sp/professionalinterest/pages/ccpr. aspx (Consulta: 24 agosto 2021).

Pacto Internacional de Derechos Económicos, Sociales y Culturales (1966) Disponible en: https://www.ohchr.org/sp/professionalinterest/pages/cescr. aspx (Consulta: 24 agosto 2021).

Ping-Kwong, K. (1996)

'Empowering Elderly People: A Community Work Approach', Community Development Journal, 31:230-240. Disponible en: https://www.jstor.org/stable/44257279 (Consulta: 24 agosto 2021). 
Protocol to the African Charter on Human and Peoples'

Rights on the Tights of Older Persons in Africa (2016) Disponible en: https:// au.int/sites/default/files/treaties/36438-treaty-0051_-_protocol_on_the_rights_of_older_persons_e.pdf(Consulta: 24 agosto 2021).

\section{Sussman v Germany (1996)}

Corte Europea de Derechos Humanos. Caso No. 20024/92.

Thomson, J. (1985)

'The Trolley Problem', Yale Law Journal, 94(6):1395-1415. DOI: 10.2307/796133. 